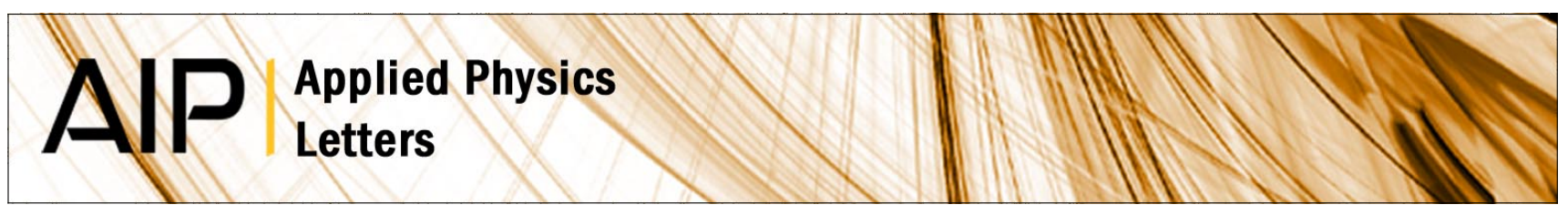

\title{
Exchange bias and memory effect in double perovskite Sr2FeCoO6
}

R. Pradheesh, Harikrishnan S. Nair, V. Sankaranarayanan, and K. Sethupathi

Citation: Appl. Phys. Lett. 101, 142401 (2012); doi: 10.1063/1.4756792

View online: http://dx.doi.org/10.1063/1.4756792

View Table of Contents: http://apl.aip.org/resource/1/APPLAB/v101/i14

Published by the American Institute of Physics.

Additional information on Appl. Phys. Lett.

Journal Homepage: http://apl.aip.org/

Journal Information: http://apl.aip.org/about/about_the_journal

Top downloads: http://apl.aip.org/features/most_downloaded

Information for Authors: http://apl.aip.org/authors

\section{ADVERTISEMENT}

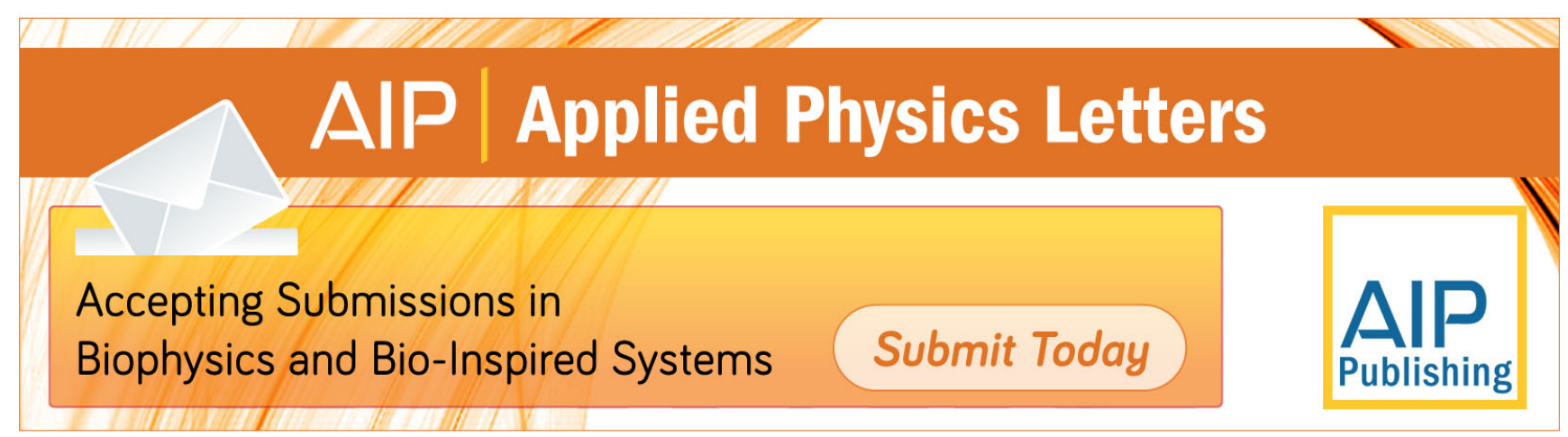




\title{
Exchange bias and memory effect in double perovskite $\mathrm{Sr}_{2} \mathrm{FeCoO}_{6}$
}

\author{
R. Pradheesh, ${ }^{1, a)}$ Harikrishnan S. Nair, ${ }^{2, a)}$ V. Sankaranarayanan, ${ }^{1}$ and K. Sethupathi ${ }^{1}$ \\ ${ }^{1}$ Low Temperature Physics Laboratory, Department of Physics, Indian Institute of Technology Madras, \\ Chennai 600036, India \\ ${ }^{2} J$ ülich Center for Neutron Sciences-2/Peter Grünberg Institute-4, Forschungszentrum Jülich, 52425 Jülich, \\ Germany
}

(Received 25 May 2012; accepted 17 September 2012; published online 1 October 2012)

We report on the observation of exchange bias (EB) and memory effect in double perovskite $\mathrm{Sr}_{2} \mathrm{FeCoO}_{6}$. Antiphase boundaries between the ferromagnetic and the antiferromagnetic regions in the disordered glassy phase are assumed as responsible for the observed effect, which reflects in the cooling field dependence and temperature evolution of exchange bias field and in training effect. The spin glass (SG) phase itself is characterized through memory, ageing, and magnetic relaxation experiments. The spin glass transition temperature, $T_{g}$, versus $H_{d c}^{2 / 3}$ follows the AlmeidaThouless line yielding a freezing temperature, $T_{f}=73 \mathrm{~K}$. Time-dependent magnetic relaxation studies reveal the magnetization dynamics of the underlying glassy phase in this double perovskite. (C) 2012 American Institute of Physics. [http://dx.doi.org/10.1063/1.4756792]

Materials that show vertical/horizontal displacement of magnetic hysteresis loop—-known as exchange bias (EB) materials - are potential candidates for technological applications as spin valves, ${ }^{1}$ permanent magnets, ${ }^{2}$ and in magnetic recording. ${ }^{3,4}$ Exchange bias is observed mainly in ferromagnetic (FM)/antiferromagnetic (AFM) bilayers ${ }^{5}$ but, also in nanoparticles, ${ }^{6}$ inhomogeneous magnets, ${ }^{2}$ and strongly correlated oxides like manganites, ${ }^{7}$ cobaltites, ${ }^{8}$ and in intermetallics. ${ }^{9}$ In classical EB systems, the hysteresis loop is shifted to the left of the origin and conventionally EB is defined negative. Positive EB has also been reported, for example, in metallic bilayers ${ }^{10}$ and in spin glasses (SGs). ${ }^{11}$ In a detailed study to distinguish between reentrant spin glass (RSG) and cluster glass (CG), exchange bias with a shift in both magnetization and field axis was observed in $\mathrm{L}_{0.5} \mathrm{Sr}_{0.5} \mathrm{CoO}_{3}{ }^{12} \mathrm{In}$ this letter, we report the observation of exchange bias in a spin glass double perovskite, thereby, extending the generality of this phenomenon.

The SG nature of $\mathrm{Sr}_{2} \mathrm{FeCoO}_{6}$, with transition temperature $T_{g} \approx 75 \mathrm{~K}$, studied through macroscopic magnetization and structural studies using neutrons has been reported elsewhere. ${ }^{13}$ In this paper, we focus on detailed magnetization measurements in field-cooled and zero field-cooled conditions along with magnetic relaxation measurements conducted using commercial SQUID magnetometer and physical property measurement system (both M/s Quantum Design Inc.).

As the first set of magnetization measurements, fieldcooled hysteresis curves at different temperatures were measured on $\mathrm{Sr}_{2} \mathrm{FeCoO}_{6}$. To this effect, the sample was field-cooled from $120 \mathrm{~K}$ to a temperature below $T_{g}$ with an applied field of $50 \mathrm{Oe}$ (after each $M(H)$ curve the sample was demagnetized by warming up to $120 \mathrm{~K}$ ). The field-cooled magnetic hysteresis loops at different temperatures in the range $30-70 \mathrm{~K}$, which show clear shifts resembling EB are presented in Fig. 1(a). The loop-shifts in the $M(H)$ plots, as seen in the figure, can signify exchange bias due to the spins

${ }^{a}$ R. Pradheesh and Harikrishnan S. Nair contributed equally to this work. at FM/AFM, FM/SG interfaces. In $\mathrm{Sr}_{2} \mathrm{FeCoO}_{6}$, antisite disorder leads to $\mathrm{SG}$ phase at low temperature which then forms FM/SG interfaces which can cause exchange anisotropy. In order to avoid minor loop effect in the observation of a genuine EB-shift, the optimal maximum applied field $\left(H_{\max }\right)$ should be greater than the anisotropy field $\left(\mathrm{H}_{A}\right)$ of the system. From the analysis of initial magnetization at $50 \mathrm{~K}$ using $M=M_{s}\left(1-a / H-b / H^{2}\right)+\chi H$ and using the

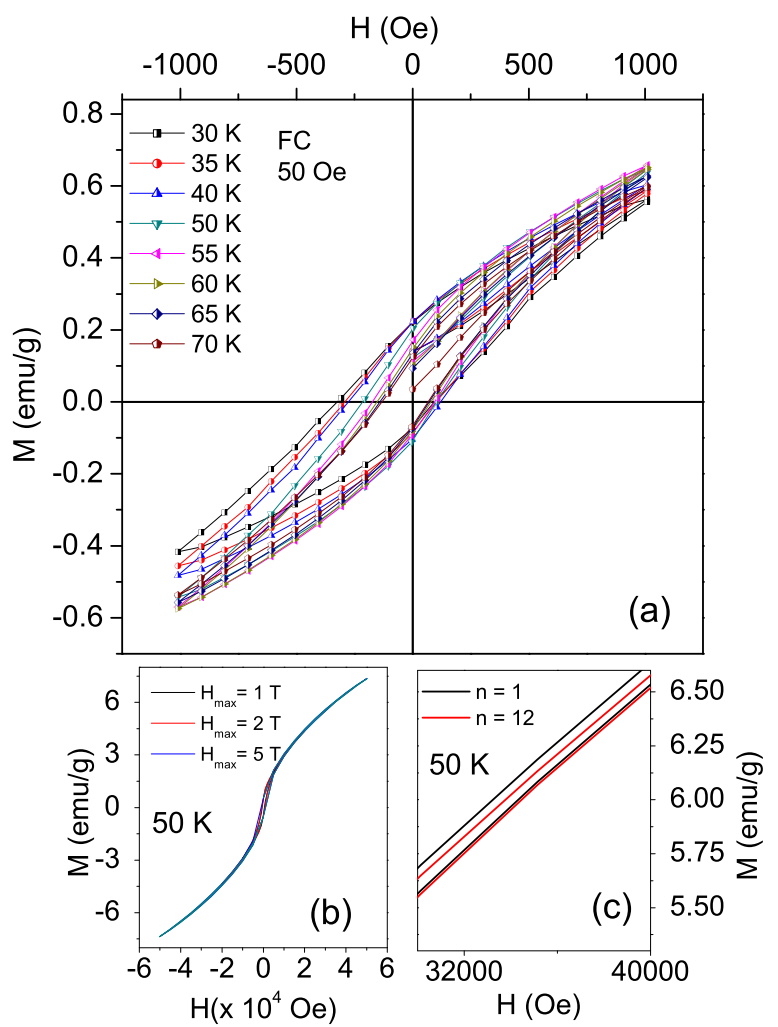

FIG. 1. (a) Field-cooled $(50 \mathrm{Oe})$ isothermal magnetization of $\mathrm{Sr}_{2} \mathrm{FeCoO}_{6}$ at different temperatures below $T_{g}$. The field range is limited to $\pm 1000 \mathrm{Oe}\left(H_{\max }\right)$, which is greater than $H_{A}$ (i.e., $\left.H_{\max }>H_{A}\right)$. (b) Hysteresis plots at different $H_{\max }$ where exchange bias disappears. (c) Training effect at $50 \mathrm{~K}$ observed with 12 loops. 
relations $b=4 K_{1}^{2} / 15 M_{s}^{2}$ and $H_{A}=2 K_{1} / M_{s},{ }^{14,15}$ a rough estimate of the anisotropy field $H_{A}=448$ Oe was obtained ( $M_{s}$ is saturation magnetization, $a, b$ are free-parameters, $K_{1}$ is anisotropy constant, $\chi$ is the high-field susceptibility). Consequently, our hysteresis measurements were performed such that the maximum applied field $H_{\max }>H_{A}$. Fig. 1(b) shows that the effect of applied fields greater than $10 \mathrm{kOe}$ is to diminish the effect of exchange bias. Similar effect of vanishing EB at high fields has been reported for cluster glass perovskite cobaltites. ${ }^{16}$ In order to confirm that the exchange bias effect is intrinsic, we performed training effect experiment where the $M(H)$ curve at $50 \mathrm{~K}$ is recorded in fieldcooled condition for 12 continuous loops. In Fig. 1(c), a magnified view of the 1 st and 12th loops is presented showing a clear shift which is typical of the response from exchange biased systems and hints at the metastable nature of the interface. ${ }^{17}$ Field-cooled hysteresis curves at $50 \mathrm{~K}$ were measured as a function of different cooling fields, $H_{F C}$. The exchange bias field was estimated from the $M(H)$ loops as $\left|H_{E B}\right|=\left|\left(H_{+}+H_{-}\right) / 2\right| ; H_{+}$and $H_{-}$are the positive and negative intercepts of the magnetization curve with the field axis. The variation of $H_{E B}$ as a function of $H_{F C}$ and temperature is shown in Figs. 2(a) and 2(b), respectively. In order to probe RSG features in the present system, we performed field-cooled hysteresis measurements at different cooling fields as suggested in Mukherjee et al. ${ }^{12}$ The resulting $M(H)$ plots are presented in Fig. 2(c). Displaced hysteresis loops are evident which with increasing $H_{F C}$ intersect the $H$-axis at a progressively higher negative values. Similar feature has been observed in $\mathrm{La}_{0.5} \mathrm{Sr}_{0.5} \mathrm{CoO}_{3}{ }^{12}$ and signifies the presence of FM clusters.

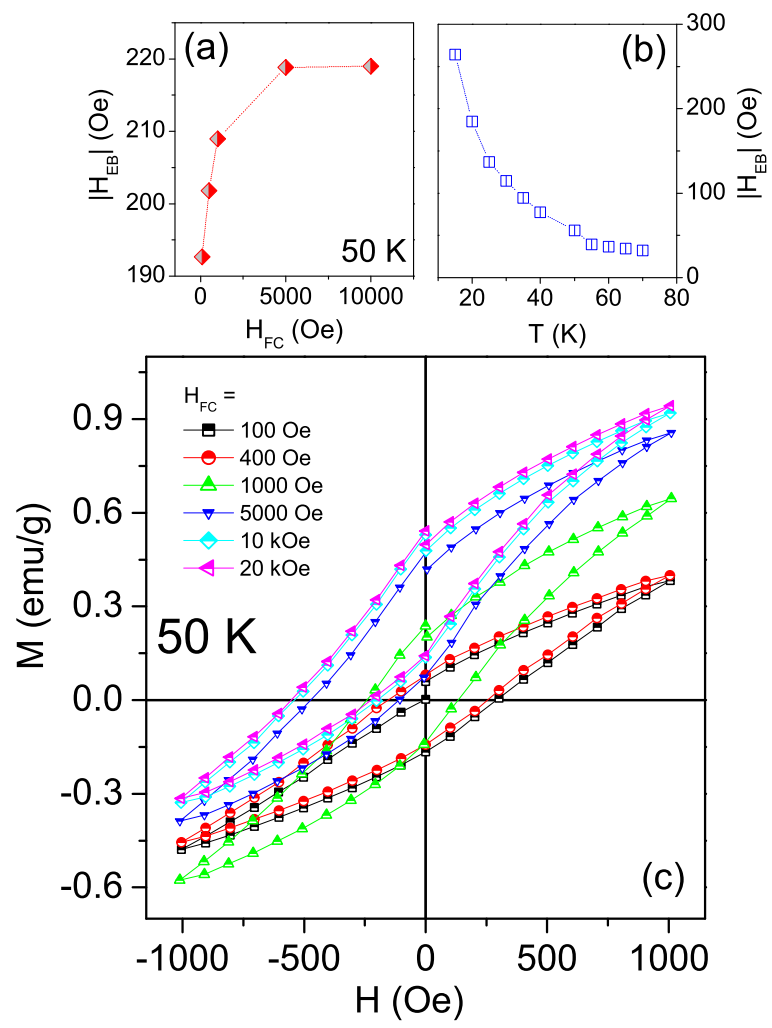

FIG. 2. Exchange bias field $H_{E B}$ plotted against (a) $H_{F C}$ and (b) temperature conform to typical exchange bias characteristics. (c) The field-cooled hysteresis curves at different cooling fields up to $20 \mathrm{kOe}$ display vertical displacement that signifies FM clusters present in the system.
In the case of spin glass systems, the peak at $T_{g}$ in the imaginary part of ac magnetic susceptibility shifts to low temperature with increasing value of superimposed dc field. The evolution of $T_{g}$ with applied magnetic field can be explained by Almeida-Thouless $(A T)$ line in a $H-T$ phase diagram. ${ }^{18}$ The $A T$ line is described by the following equation:

$$
\frac{H_{d c}(T)}{\Delta J}=\left(1-T_{g} / T_{C}\right)^{\alpha}
$$

Here, $\Delta J$ is the width of the distribution of exchange interactions, $H_{d c}$ is the superimposed dc magnetic field, and $\mathrm{T}_{C}$ is the transition temperature. Fig. 3(a) is the plot of $T_{g}$ versus $H_{d c}^{2 / 3}$ for $\mathrm{Sr}_{2} \mathrm{FeCoO}_{6}$, which shows a decrease in $T_{g}$ with applied field. A fit to the variation of $T_{g}$ with field assuming the critical exponent $\alpha=3 / 2$ gives straight line fit satisfying the $A T$ equation and confirms the spin glass nature of $\mathrm{Sr}_{2} \mathrm{FeCoO}_{6}$. Linear behaviour with the critical exponent being $3 / 2$ is observed for low fields $\left(\mu_{0} \mathrm{H}<1 \mathrm{~T}\right)$. Extrapolating the fit to both the axes, we obtain the freezing temperature $\left(T_{f}\right)$ and the critical field $\left(H_{c r}\right)$ as $73 \mathrm{~K}$ and $116 \mathrm{Oe}$, respectively. The conformity with $A T$-line has been observed in intrinsically exchange biased $\mathrm{Zn}_{x} \mathrm{Mn}_{3-x} \mathrm{O}_{4}$ solid solutions. ${ }^{19}$ For reentrant spin glass systems, the disorder extends to the whole volume resulting in the shift of $T_{f}$ to lower temperatures with increase in magnetic field. ${ }^{20}$

In order to further test the glassy magnetic ground state in $\mathrm{Sr}_{2} \mathrm{FeCoO}_{6}$, memory experiments were conducted, for which, the sample is first zero field-cooled to low temperatures at a constant cooling rate, while recording magnetization. While cooling, intermediate stops are administered below $T_{c}$ when the measurement is stopped (for $2 \mathrm{~h}$ ) and

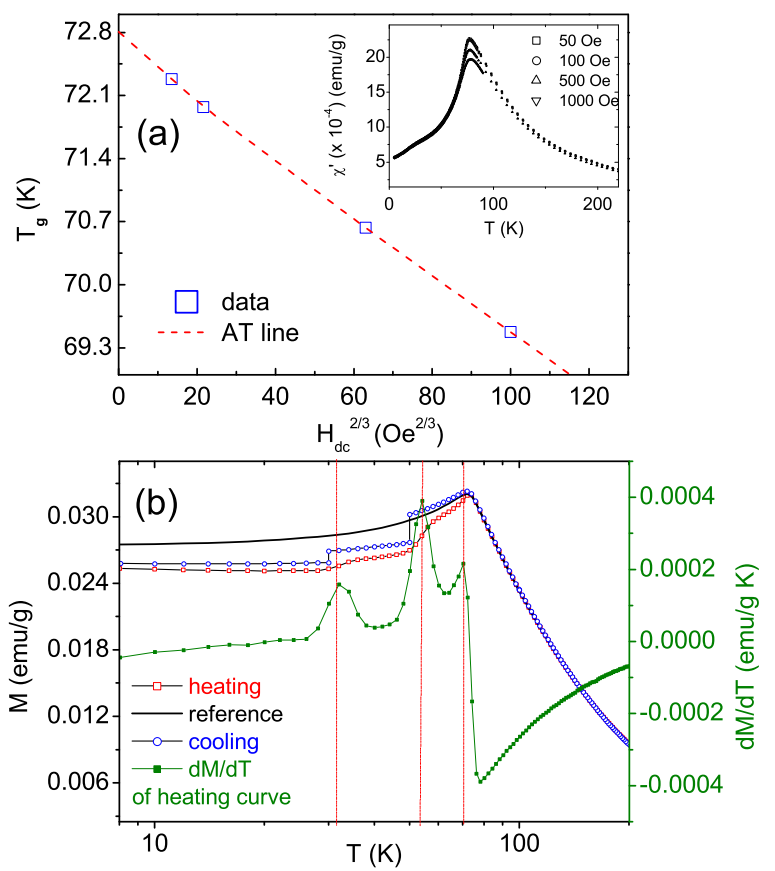

FIG. 3. (a) $T_{g}$ vs $H_{d c}^{2 / 3}$ plot for $\mathrm{Sr}_{2} \mathrm{FeCoO}_{6}$ where $H_{d c}$ is the superimposed dc field and the dashed-line shows the fit according to Eq. (1). The inset shows the real part of ac susceptibility, $\chi^{\prime}(T)$, at different applied dc fields. (b) Memory effect in $\mathrm{Sr}_{2} \mathrm{FeCoO}_{6}$. The stops administered during the cooling curve, where the measurement is halted, are recovered in the heating cycle. The derivative plots clearly show the recovered stops. For comparison, a reference measurement curve (without stops) is also presented. 
magnetization is allowed to relax. After reaching the lowest possible temperature, the sample is heated back at a constant heating rate without administering any stops and magnetization is recorded. For comparison, a reference curve where no stops are administered is also recorded. The cooling, heating, reference and the derivative of the heating curve, $d M / d T$, are presented in Fig. 3(b). Clear "dips" in magnetization of the cooling curve are discernible, where the measurement was stopped. The signatures of memory effect are clear in the heating cycle where the steps are recovered at the same temperature points. This is clearly visible in the plot of $d M / d T$. Observation of memory effect is a confirmation of the magnetic glassy state and has been reported in canonical spin glasses and phase separated manganites that show spin glasslike ground states.

In order to study the magnetic relaxation mechanisms stemming from the underlying magnetic glassy state, time dependent magnetization with different wait times was recorded at $50 \mathrm{~K}$. For these measurements, the sample was zero field-cooled to $50 \mathrm{~K}$, a wait time $t_{w}$ was administered, and then the magnetization was measured as a function of time. Evident from Fig. 4(a), a clear dependence on $t_{w}$ can be seen wherein the system becomes magnetically stiffer as the wait time increases; which is common among canonical spin glasses. Fig. 4(b) shows time-dependent magnetization at $t_{w}=3600 \mathrm{~s}$ but at different temperatures. The timedependent magnetization was fitted well with a stretched exponential of the form

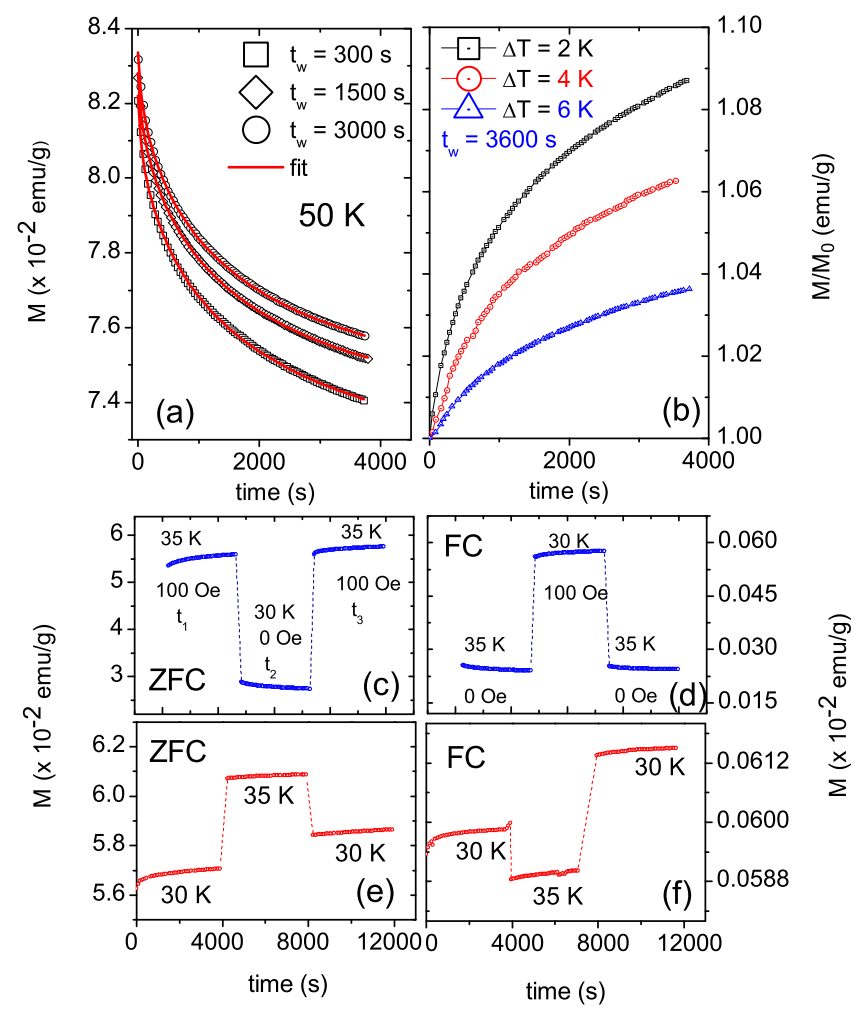

FIG. 4. (a) Time-dependent magnetization of $\mathrm{Sr}_{2} \mathrm{FeCoO}_{6}$ at $50 \mathrm{~K}$ for three different wait times. The solid lines are fit to the Eq. (2). (b) The timedependent magnetization with a wait time of $t_{w}=3600 \mathrm{~s}$. Signatures of aging are present in relaxation experiments in both ZFC ((c) and (e)) and FC ((d) and (f)) protocols. (c) and (d) show negative temperature cycling in ZFC and FC modes, respectively, while (e) and (f) show the same plots in warming mode. Asymmetric response is clearly seen for the warming mode.

$$
M(t)=M_{0}-M_{g} \exp \left[-\left(\frac{t}{t_{r}}\right)^{1-n}\right]
$$

where $M_{0}$ is the intrinsic ferromagnetic moment, $M_{g}$ is the glassy component of the moment, $t_{r}$ is the characteristic time component, and $n$ is the stretched exponential exponent. Equation (2) is similar to Kohlrausch law ${ }^{21}$ which is used to explain magnetic, dielectric, and optical phenomena, where relaxation mechanisms play an important role in the dynamics. The exponent in Eq. (2) is temperature dependent, and according to the percolation model, varies in the range $\frac{1}{3} \leq n \leq 1 .^{22}$ Table I shows the parameters obtained from the fit according to Eq. (2). The values of $n$ and $M_{0}$ are independent of $t_{w}$ while the characteristic time scale $t_{r}$ varies with $t_{w}$ typical of canonical spin glasses. ${ }^{23}$ The characteristic time varies slowly with the wait time, but the dependence of $t_{r}$ on $t_{w}$ implies that it is in a non-equilibrium state and that of the memory effect. Negative temperature cycling of magnetization in zero field-cooled (ZFC) and field-cooled (FC) protocols was also performed to complement the memory effect. In the ZFC protocol, the sample was cooled down to $35 \mathrm{~K}$ in ZFC mode and a field of 100 Oe was applied to measure magnetization for time $t_{1}$. Further, the sample was cooled down to $30 \mathrm{~K}$, the field is switched off, immediately after which the magnetization is recorded for another time $t_{2}$. After $t_{2}$, the system is taken back to $35 \mathrm{~K}$ and magnetization measured for time $t_{3}$. In the above measurement, $t_{1}=t_{2}=t_{3}=3600 \mathrm{~s}$. Fig. 4(c) shows the ZFC temperature cycling where the effect of memory is observable even after aging at lower temperature. Fig. 4(d) illustrates the same experiment as in (c) but, in FC protocol. Similar measurements (ZFC and FC) but in heating cycle were performed the results of which are given in Figs. 4(e) and 4(f). An asymmetric response is observed which means that there is no memory while heating the sample.

The relaxation measurements confirm the spin glass nature of $\mathrm{Sr}_{2} \mathrm{FeCoO}_{6}$ and can be concluded that the observed exchange bias is seen in the spin glass phase. Exchange bias systems with $\mathrm{FM} / \mathrm{SG}$ interfaces are known to show exponential decrease in $H_{E B}$ with temperature. ${ }^{7}$ In such a scenario, the SG phase forms the frozen phase where magnetization is irreversible while that of the FM phase is reversible. In the present case, we assume a minority FM phase which coexists in a majority AFM disordered (glassy) phase as deduced from the hysteresis curves and lack of saturation magnetization. With reduction in temperature, increasing number of disordered AFM (or glassy) domains freeze and this progressive freezing leads to enhancement of $H_{E B}$ at low temperature. The dependence of $H_{E B}$ on $H_{F C}$ is understood based on the competition between the Zeeman coupling energy and

TABLE I. The parameters, $M_{0}, M_{g}, t_{r}$, and $n$ obtained by fitting the timedependent magnetization at different wait times using Eq. (2).

\begin{tabular}{llccc}
\hline \hline$t_{w}(\mathrm{~s})$ & $M_{0}(\mathrm{emu} / \mathrm{g})$ & $M_{g}(\mathrm{emu} / \mathrm{g})$ & $t_{r}(\mathrm{~s})$ & $n$ \\
\hline 300 & $0.009(3)$ & $0.074(1)$ & $1590(1)$ & $0.43(1)$ \\
1500 & $0.009(2)$ & $0.073(2)$ & $1671(2)$ & $0.41(2)$ \\
3000 & $0.0102(3)$ & $0.071(2)$ & $1705(1)$ & $0.41(5)$ \\
\hline \hline
\end{tabular}


the exchange energies at the interfaces. At low cooling fields, field-cooling induces progressive enhancement of freezing of domains and hence $H_{E B}$ increases, whereas at high cooling fields, Zeeman energy overcomes the magnetic interactions at the interface.

In conclusion, we report the observation of exchange bias in the double perovskite $\mathrm{Sr}_{2} \mathrm{FeCoO}_{6}$ which is a spin glass. The claim of intrinsic exchange bias is supported through field-cooled hysteresis measurements, dependence of exchange bias fields on cooling fields and temperature, training effect, etc. Interface magnetic interactions between FM regions and SG domains are believed to be the origin of observed exchange bias. The underlying spin glass phase is further characterized through the conformation with AT-line, memory and aging effects, and magnetic relaxation.

The authors acknowledge the Department of Science and Technology (DST), India for the financial support for providing the facilities used in this study (Grant No. SR/ FST/PSII-002/2007) and (Grant No. SR/NM/NAT-02/2005).

${ }^{1}$ B. G. Park, J. Wunderlich, X. Martí, V. Holý, Y. Kurosaki, M. Yamada, H. Yamamoto, A. Nishide, J. Hayakawa, H. Takahashi et al., Nature Mater. 10(5), 347 (2011).

${ }^{2}$ J. Nogués and I. K. Schuller. J. Magn. Magn. Mater. 192, 203 (1999).

${ }^{3}$ J.-W. Liao, R. K. Dumas, H.-C. Hou, Y.-C. Huang, W.-C. Tsai, L.-W. Wang, D.-S. Wang, M.-S. Lin, Y.-C. Wu, R.-Z. Chen, C.-H. Chiu, J. W. Lau, K. Liu, and C.-H. Lai, Phys. Rev. B 82(1), 014423 (2010).

${ }^{4}$ I. V. Roshchin, O. Petracic, R. Morales, Z. P. Li, X. Batlle, I. K. Schuller, U.S. patent 7,764,454 (2010).
${ }^{5}$ M. D. Stiles and R. D. McMichael, Phys. Rev. B 63, 064405 (2001).

${ }^{6}$ V. Skumryev, S. Stoyanov, Y. Zhang, G. Hadjipanayis, D. Givord, and J. Nogués, Nature 423(6942), 850 (2003).

${ }^{7}$ S. Karmakar, S. Taran, E. Bose, B. K. Chaudhuri, C. P. Sun, C. L. Huang, and H. D. Yang, Phys. Rev. B 77, 144409 (2008).

${ }^{8}$ Y.-k. Tang, Y. Sun, and Z.-h. Cheng, Phys. Rev. B 73, 174419 (2006).

${ }^{9}$ X. H. Chen, K. Q. Wang, P. H. Hor, Y. Y. Xue, and C. W. Chu, Phys. Rev. B 72, 054436 (2005).

${ }^{10}$ J. Nogués, D. Lederman, T. J. Moran, and I. K. Schuller, Phys. Rev. Lett. 76, 4624 (1996)

${ }^{11}$ M. Ali, P. Adie, C. H. Marrows, D. Greig, B. J. Hickey, and R. L. Stamps, Nature Mater. 6, 70 (2007).

${ }^{12}$ S. Mukherjee, R. Ranganathan, P. S. Anilkumar, and P. A. Joy, Phys. Rev. B 54(13), 9267 (1996).

${ }^{13}$ R. Pradheesh, Harikrishnan S. Nair, C. M. N. Kumar, J. Lamsal, R. Nirmala, P. N. Santhosh, W. B. Yelon, S. K. Malik, V. Sankaranarayanan, and K. Sethupathi, J. Appl. Phys. 111(5), 053905 (2012).

${ }^{14}$ S. V. Andreev, M. I. Bartashevich, V. I. Pushkarsky, V. N. Maltsev, L. A. Pamyatnykh, E. N. Tarasov, N. V. Kudrevatykh, and T. Goto, J. Alloys Compd. 260(1), 196-200 (1997).

${ }^{15}$ M. Patra, M. Thakur, K. De, S. Majumdar, and S. Giri, J. Phys.: Condens. Matter 21, 078002 (2009).

${ }^{16}$ W. Luo and F. Wang, Appl. Phys. Lett. 90, 162515 (2007).

${ }^{17}$ S. Giri, M. Patra, and S. Majumdar, J. Phys.: Condens. Matter 23, 073201 (2011).

${ }^{18}$ J. R. L. de Almeida and R. Bruinsma, Phys. Rev. B 35, 7267 (1987).

${ }^{19}$ D. P. Shoemaker, E. E. Rodriguez, R. Seshadri, I. S. Abumohor, and T. Proffen, Phys. Rev. B 80, 144422 (2009).

${ }^{20}$ B. Martinez, X. Obradors, Ll. Balcells, A. Rouanet, and C. Monty, Phys. Rev. Lett. 80(1), 181 (1998).

${ }^{21}$ R. G. Palmer, D. L. Stein, E. Abrahams, and P. W. Anderson, Phys. Rev. Lett. 53(10), 958 (1984).

${ }^{22}$ R. Böhmer, K. L. Ngai, C. A. Angell, and D. J. Plazek, J. Chem. Phys. 99(5), 4201 (1993).

${ }^{23}$ R. V. Chamberlin, Phys. Rev. B 30(9), 5393 (1984). 